\title{
Using system mapping to help plan and implement city-wide action to promote physical activity
}

\author{
Nick Cavill, ${ }^{1}$ Debra Richardson, ${ }^{2}$ Mark Faghy, ${ }^{2}$ Chris Bussell, ${ }^{2}$ Harry Rutter ${ }^{3}$ \\ ${ }^{1}$ Cavill Associates Ltd., and Centre for Exercise, Nutrition \& Health Sciences, School for Policy Studies, \\ University of Bristol; ${ }^{2}$ Human Sciences Research Centre, College of Life and Natural Sciences, University of \\ Derby; ${ }^{3}$ Department of Social and Policy Sciences, University of Bath, UK
}

\begin{abstract}
Background: There is growing interest in conceptualising public health problems such as physical inactivity as the outcome of multiple interacting factors within complex systems. A systems approach includes consideration of the indirect as well as direct impacts of interventions, the contexts within which they take place, the relations between the multiple factors at play, and the ways in which systems adapt in response to changes. This study involved working with stakeholders involved in a city-wide physical activity promotion programme in Derby, UK to investigate whether a conceptual map of the local physical activity system could be a useful tool to help improve the planning and implementation of the programme.

Methods: We produced draft conceptual maps of the major modifiable drivers of physical activity in the city, based on the existing literature on determinants and correlates of physical activity, then refined them in a series of stakeholder meetings. The maps were used to explore ways in which the existing programme took a systems approach, and how it might be enhanced. Semistructured interviews were subsequently undertaken with stakeholders to assess their views on the contribution of the mapping approach.

Results: Feedback from stakeholders described the mapping as valuable, especially in helping to identify the limitations of the original approach taken in the city.

Conclusions: Even a very simple application of systems thinking can be a useful tool for disaggregating the key factors driving a system, helping to identify areas that merit greater attention, and supporting effective action.
\end{abstract}

\section{Introduction}

Regular physical activity provides many important health benefits. ${ }^{1}$ Physical activity (PA) promotion is an important component of public health approaches to the prevention of non-communicable diseases (NCDs) and maintenance of functional capacity and quality of life into older age. ${ }^{2}$ However, public health and associated strategies and investment to date have not had a significant influence on population levels of activity, which remain low in most countries. ${ }^{3,4}$

The evidence for the health and associated social and economic benefits of PA is extremely strong. However, the evidence base remains weak for effective strategies to achieve the long-term increases in activity levels across the life course that are required to achieve population-level improvements in health and other important outcomes. ${ }^{5}$ Although evidence on effective approaches for increasing PA among individuals or groups exists, the literature is dominated by studies on short-term, small-scale, individual-level interventions, with significant challenges remaining in how to implement at scale. ${ }^{6}$

A growing appreciation of this problem has helped to drive an increasing emphasis on approaches to increasing PA that operate at multiple 'levels' and/or in multiple domains, tackling a broad range of determinants of PA behaviour. The socio-ecological model addresses multiple influences on an individual's health, ${ }^{7}$ from personal factors through to the socio-political system, and it has been used to help plan interventions at multiple levels. ${ }^{8,9}$ For example, in the UK a number of multi-level community-wide approaches based on the socio-ecological model have been used to promote PA, such as Local Exercise Actions Pilots ${ }^{10}$ and Cycling Demonstration Towns. ${ }^{11}$ These approaches to promoting PA recognised the varied influences on health and implemented a range of interventions to tackle them. This included using behavioural interventions - such as those that encourage people to consider the merits and demerits of becoming more active in order to support them in developing their own personal strategies alongside efforts to make the physical environment more amenable to PA, while also attempting to change social norms around being active. However, to date, these approaches have paid little regard to the potential for interactions between the different 'levels' of intervention ${ }^{12}$ or the drivers of the structural factors that influence physical activity, and have tended to be centrally-administered programmes that implemented standardised approaches irrespective of the nature of the local PA system.

Significance for public health

This paper has shown the value of using systems mapping to help stakeholders interested in complex public health problems take a broader view of the topic. This includes disaggregating the key factors driving a system, helping to identify areas that merit greater attention, and supporting effective action. This approach can be applied to any complex public health problem. 
More recently, there has been an increasing focus on considering public health challenges - including physical inactivity - as the outcome of multiple interacting factors within complex systems. This came to prominence in the UK with the UK Government Foresight report on obesity ${ }^{13}$ which introduced the concept of complex systems thinking to a broad public health audience. Complex systems can be considered as a collection of elements (e.g., subsystems, sectors), with interconnections between those elements, ${ }^{12}$ and other characteristics including feedback, non-linearity, adaptation, and emergence. ${ }^{14}$ Systems approaches emphasise the importance of studying a whole system and its dynamic relations, rather than single elements in isolation. ${ }^{12}$ For example, a traditional evaluation of a programme aimed at encouraging families to walk their children to school might focus primarily on the impact on rates of walking. A systems approach, however, might identify a number of indirect but potentially important impacts such as changes to social norms; improved road safety; reduced acceptability of car driving; reductions in carbon emissions and other pollutants affecting air quality; and improved social interaction. An appreciation of these wider and connected impacts, and the relations between them may lead to a more nuanced understanding of the factors that might influence PA across the local system. ${ }^{15}$

\section{The role of systems maps}

A useful first step in exploring complex systems is to produce a conceptual 'map' of the multiple factors that constitute the system being considered and the ways that they interact. This can have a number of functions, including communicating multiple factors and the cross-sectoral nature of the influences on health outcomes for policymakers; illustrating the breadth of opportunities to influence the system; strengthening partnerships, and identifying key areas for action that may represent significant policy leverage. ${ }^{15}$ These maps can be developed in close consultation with the community affected ${ }^{16,17}$ and may include contributions from expert stakeholders. ${ }^{12}$

This study built on an approach described by Allender et al. ${ }^{16}$ who worked with communities in Australia to develop causal loop diagrams based on community perceptions of determinants and causes of obesity. These causal loop diagrams (CLDs) show the key determinants or influences on an outcome as nodes on the map, with the connections between them shown as lines. The aim of this project was to investigate whether systems mapping could be a useful tool to help improve the planning and implementation of a city-wide PA programme, through promoting the use of systems thinking.

\section{Derby: a City on the Move - a population approach to promoting physical activity}

Efforts to promote PA at town or city-wide level provide a natural geographical focus, with the administration of public infrastructure usually controlled by a local government agency, who can therefore make changes to local environments to promote activity. There are several examples of town-wide or area-wide approaches to PA promotion, from international case studies of pioneering cardio-vascular disease prevention programmes ${ }^{18,19}$ through to more recent initiatives..$^{10,11,20-22}$ These studies have tended to take a multi-level rather than a systems approach, with the exception of the Healthy Towns programme in the UK, which was partly informed by systems thinking. ${ }^{23}$ More recently, Sport England (the government agency responsible for promoting sport and PA in England) has announced funding for city-wide programmes to promote PA using a 'whole systems approach'. ${ }^{24}$ One such project is 'Derby: a City on the Move (DaCotM)' which began as an ambitious partnership approach to tackle physical inactivity for those who live, work or study in Derby, UK. The project was funded until December 2018 through Sport England's 'Get Healthy, Get Active' funding stream and through in-kind contributions from a consortium of partners that included higher and further education establishments, community trust organisations and the local government. The original vision for DaCotM was to achieve sustained changes in PA behaviour through the adoption of what has been described as a 'whole systems approach'. Evaluation methods for the programme included quantitative and qualitative data collection and analysis, focusing primarily on changes in PA status of inactive individuals accessing the programme. DaCotM was chosen for this study as it was conducted at a citywide level; was focused on PA; had set out to take a systems approach; and had not previously explored the potential role of systems mapping.

\section{Methods}

The initial phase of this project focused on mapping the factors influencing PA in the city, and using this map to explore relations between the programme and the existing PA system. There was insufficient time in the project to generate a map with stakeholders from scratch, so in order to expedite the process a draft map was produced (by $\mathrm{NC}$ and $\mathrm{HR}$ ), based on the existing literature on determinants and correlates of PA. ${ }^{25-27}$ This draft map was presented to stakeholders who then amended it to reflect the specific characteristics of the local context.

A half-day workshop was held with key local stakeholders from the DaCotM consortium including local government organisations, registered charities and further and higher education providers. Following an introductory session that explained the conceptual basis of systems approaches to PA promotion, the initial draft map was presented to the stakeholders. They were asked to consider if there were any important factors omitted from the map and if any of the elements of the map should be amended or removed. This led to a number of changes being made and resulted in a revised map (Figure 1). Stakeholders then considered three core questions:

i. What are the main areas of action for the Derby: a City on the Move programme?

ii. What are the main types of action shown on the map that are likely to make a material difference to population levels of physical activity in Derby?

iii. What data are already being collected that could be used to measure the impact of the programme?

Following a discussion of these questions, the systems map was then re-drawn to include the additional nodes and connections that had been proposed by the group, and these revised maps were then taken to a second meeting. This was attended by a broader group of stakeholders, based on recommendations from the first workshop. The focus of this meeting was i) to discuss the actions that had been identified and where they were located on the map, and ii) to identify the data that were being collected across the PA system and how they might inform the evaluation of the programme. These questions were also explored in subsequent telephone interviews with stakeholders.

Semi-structured interviews were conducted in May 2018, approximately six months after the meetings in which the systems maps had been drafted and discussed. The interviews focused on the process of mapping and discussion; how useful or otherwise people had found the process; whether it provided direction for their programme; and ideas for changes to the process if it were conducted again. The interviews were undertaken with a convenience sample of six of the key stakeholders who had been 
involved in the process. These stakeholders were selected as people who had been engaged in the mapping process; had been involved in strategy development across the City; and represented a range of different professional sectors (academia; transport; sport; local government). Interviews were approximately $30 \mathrm{~min}$ long and conducted using a discussion guide. They were recorded and transcribed verbatim. Transcripts were analysed and coded by one researcher (NC) with a sample checked by another (HR). Meeting notes and written comments from the mapping sessions (approximately 12-15 attendees per session) were used to corroborate the findings from the interviews where possible. Thematic analysis of all the data focused on the key themes emerging with an a priori focus on the usefulness of the mapping process for programme planning and implementation. In keeping with the collaborative nature of this project, three of the interviewees were subsequently invited to become co-authors on this paper (DR, CB and MF).

Ethical approval for this research was obtained from the Human Science Ethics Committee at the University of Derby in advance of the interviews. Written informed consent to participate was obtained from all participants, who were informed that their responses would be published.

\section{Results}

The feedback from the initial meeting group (approx. 12 attendees) identified a number of minor revisions to be made to the draft map; these are highlighted in Figure 1. The resulting map shows what the stakeholders present in that meeting regarded as the main high-level influences that operated on the outcome of total PA in Derby, as well as on three specific domains of activity: walking for transport; cycling for transport, and sport and active recreation.

Figure 2 presents the main areas of activity within DaCotM identified by the stakeholders. This shows that the primary focus had been on individual level behaviour change, through the provision of information and social marketing campaigns.

This analysis underlined three key issues:

1. Stakeholders highlighted that their programme had been primarily focused on the interpersonal domain, shown on the right-hand side of the systems map;

2. Stakeholders agreed that the built and natural environments had a key influence on PA (shown primarily on the left of the systems map) but the programme had not been designed to incorporate environmental interventions up to that point;

3. Stakeholders stated that changing social norms was a key aim of the programme but analysis of the systems map showed that there had been little activity pursuing this aim to date.

Finally, stakeholders discussed data collection (Figure 3). Discussions identified a number of issues:

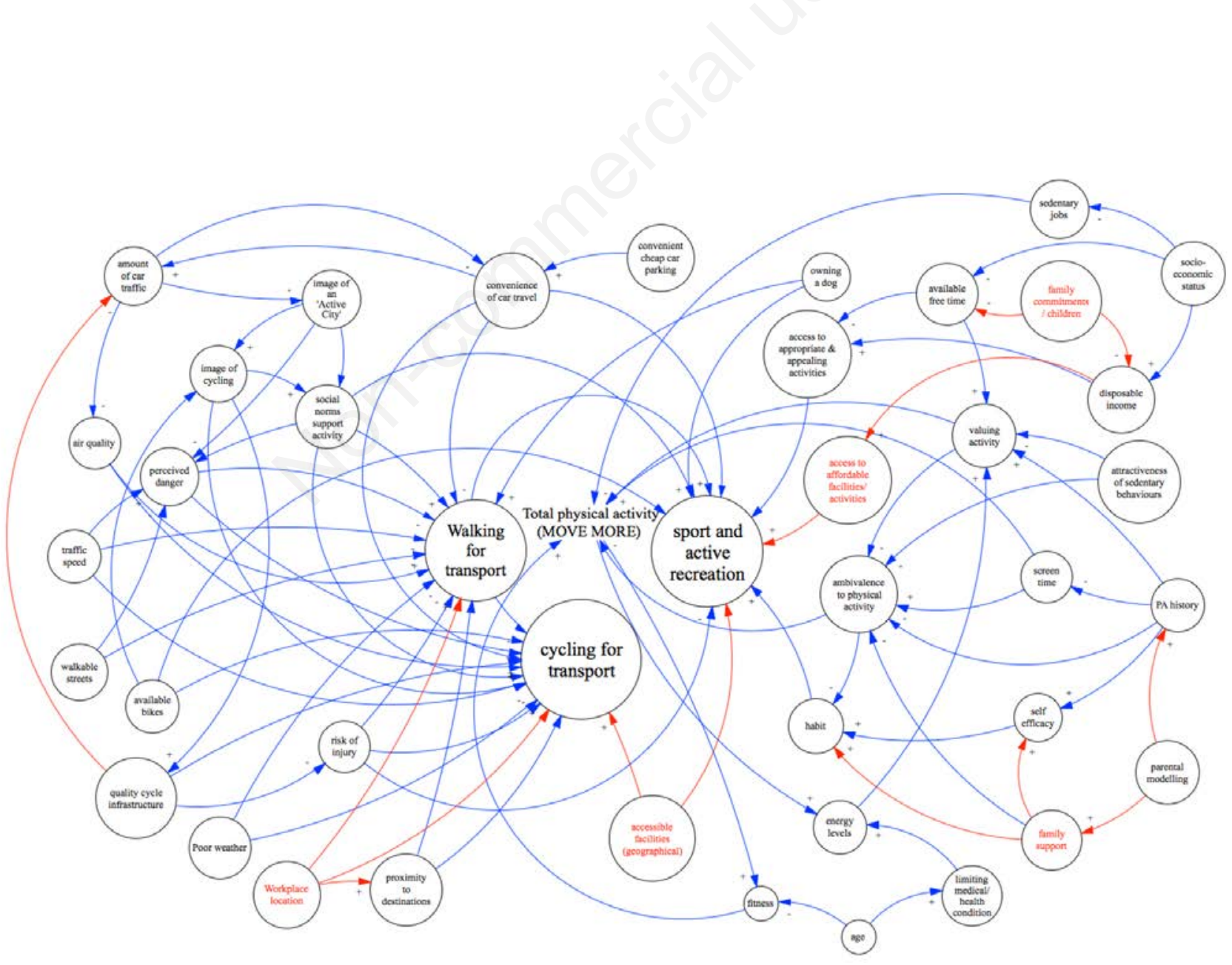

Figure 1. Initial system map (shown in black and blue) with additions made at the first meeting (highlighted in red). 
1. The programme was collecting core data on PA participation using surveys. It had set up data collection systems using routine data (such as the Active Lives Survey ${ }^{28}$ to monitor changes in the main outcomes of the programme: PA participation (and participation in the specific modes of activity such as sport and active travel);

2. The programme was also collecting some data on attitudes and barriers to PA but this was primarily among people attending specific PA sessions and was not at a population level;

3. Despite having identified social norms around PA as a key issue in the city, no data were being collected on social norms around PA, or on awareness of the programme;

4. Stakeholders reported that there was a wealth of data on environmental and transport issues that were being collected routinely by the local authority, but these were not currently available to or used by the programme.

Following this analysis, recommendations were made to i) broaden the programme; ii) take more of a systems approach in the future work of the programme, and iii) consult with local authority colleagues to identify a wider set of indicators.

\section{Interviews}

Interviewees were all extremely positive about the experience. All interviewees said the development and use of the maps had been worthwhile and interesting; the only substantive negative comments were that several interviewees wished the systems work had been done sooner, in more depth, and involving more people.

If we hadn't gone through this process we wouldn't have been in a position to inform some of the direction of that group ... the overarching message that should come through is that we as partners across the city want to achieve a whole system approach ... without this process I am pretty sure that would not have come through

A number of stakeholders said that the process had helped them to see the 'bigger picture'. As professionals involved in PA promotion they were aware on a basic level that there was a multitude of influences on behaviour that were interrelated, but each of them tended to work in their own 'silos'. The mapping process appeared to have helped break down some of the barriers between these silos:

I think what this process has done is increase the awareness that ...people need to step outside of what they are doing traditionally and look at things slightly differently.

The diagrams are really, really useful because it starts to build up a conversation which ... clarifies some thinking around the system and actually the inter-dependencies of it...

A related aspect is the extent to which the process helped

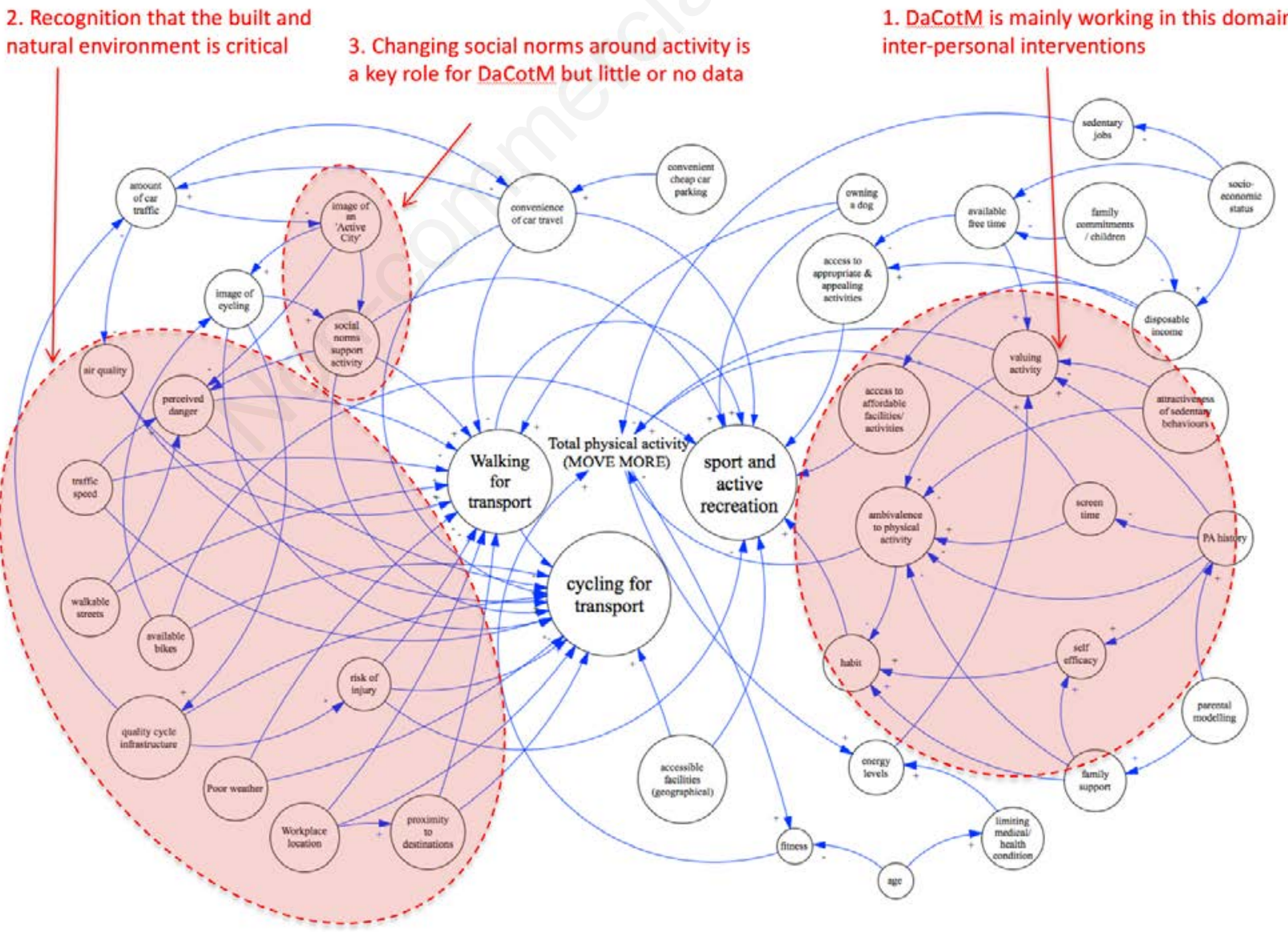

Figure 2. Initial analysis of the system map. 
stakeholders to see and begin to understand the complexity within the local PA system. Respondents reported that they found the complexity approach useful in helping them to see a wider variety of perspectives:

Until I had been involved in it I didn't appreciate the complexity of how it could all come together and how the things that I hadn't necessarily considered...

Often people throw the term ['whole system approach'] around without really understanding it. Now...there is a lot of understanding.

Some stakeholders said that while they may have found the overall systems map a little daunting at first, it increased their understanding of the role that they played in the wider system, and helped them to see themselves as 'part of the solution':

In a way it was helpful for them to see ...that they were an important part of this...they have a very vital role to play in the areas that they work in but they are part of a very big picture... and this process really helped to show that and was very, very clear in identifying ... what else need to be looked at and was very important...

\section{Key players}

One of the most tangible findings was that the stakeholders recognised that in order to adopt a systems approach, a broader range of partners needed to be engaged.

It has brought home the stark reality of how many gaps there are in this...we are still some keys players short in this

Some of the mapping you have done illustrates where some of those gaps are and how we need to bring in different agencies both from an evaluation, monitoring and intervention perspective

In particular, it was seen that there was insufficient representation from the transport and environment departments of the local authority. As a result, a number of people from other disciplines such as transport and planning were invited to join the consortium shortly after the meeting, and the PA programme was broadened and rebranded as Move More Derby. The mapping and consultation process was also seen to be helpful in terms of broadening the range of data that might be considered to be relevant to PA promotion. For example, the map prompted discussions about the importance of the quality of parks and green spaces, and further investigation showed that the local authority regularly collected data on public perception of local parks. This indicator had not been seen previously to be a part of the PA system, but stakeholders said they now saw that a wider view of the PA system also implied a wider view of what constituted relevant data:

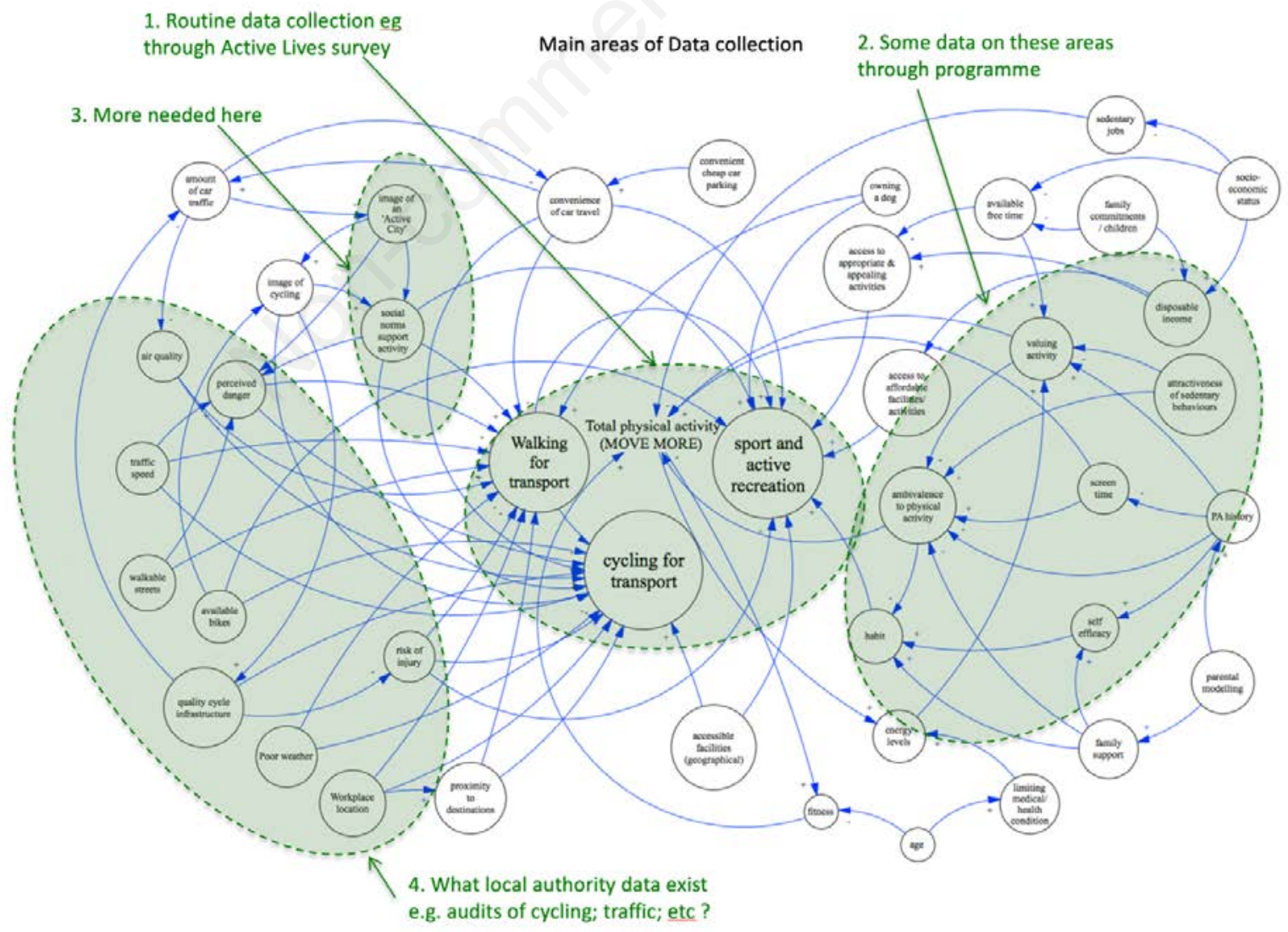

Figure 3. Initial analysis of the data collection systems. 
Looking at the sheer volume of data recording different things...there is a huge potential resource of data that we as a city and as a partnership we can potentially tap into and inform interventions or population-wide services...that is something that has been really helpful

\section{Potential improvements}

Interviewees were asked whether aspects of the mapping and development process could be improved. There was a very strong feeling among most participants that the main drawback was that insufficient numbers of people had been involved. Consulting a broader range of stakeholders was felt to be important to ensure the widest variety of perspectives:

The only thing I would say about the process that it did do in hindsight is that we probably needed more people involved in that conversation

You can almost see two half days workshop where you've got 60 or 70 people doing that process and taking it and refining it some more so that we actually have a fuller picture of the system.

\section{Discussion}

Physical activity is a behaviour that is influenced in complex ways by a wide variety of determinants, from individual to socioeconomic factors. Like many other public health issues, it lends itself to a systems approach, bringing together stakeholders from a range of sectors and disciplines to identify and then implement coordinated actions across the system.

A number of case studies where systems approaches were used to address public health problems have been described previously. ${ }^{12,16,17}$ However, these examples used more sophisticated approaches than the one we applied here. This project took a much simpler approach that focused primarily on the elements within the system, and deliberately did not attempt rigorous identification of relations between factors, leverage points, or feedback loops. Nevertheless, feedback from stakeholders showed that even this simple approach of disaggregating the key factors driving a system and helping to highlight areas that might merit greater attention was seen to have been valuable, especially in helping to show the limitations of the approach that had previously been taken in the city.

\section{Limitations of this study}

This study took a deliberately simple, pragmatic approach, with the aim of producing practical and actionable recommendations for changes to the programme. However, even within this context there are a number of ways in which it could have been improved. Firstly, more feedback from a wider range of stakeholders would have added to the strength of the findings, and their credibility and influence within the local system. Secondly, it would have added strength to the study to add further interviews at a later date to explore longer-term changes to the programme.

The maps produced are relatively basic in their design, and we have also subsequently developed the mapping techniques from these early approaches, including use of new mapping software that allows for more explicit construction of putative causal pathways and feedback loops.

\section{Conclusions}

This study shows that even a simplified approach to systems mapping can help to develop systems thinking among stakeholders, and may be a welcome approach to help people conceptualise the broad context in which a public health programme operates. This approach thus has important potential for use in other towns and cities where public health challenges require responses across systems. Future developments could include taking a more sophisticated approach to develop causal loop diagrams, using them as tools to identify and explore feedback loops and potential levers ${ }^{29}$ for influencing the PA system over the short, medium and long term, and then applying this learning to develop more effective and sustainable interventions.

Correspondence: Nick Cavill, 18 Fords Lane, Bramhall, Stockport SK7 1DQ UK. E-mail: nick@cavill.net

Key words: physical activity; complex systems; mapping; intervention planning.

Acknowledgements: The authors would like to thank Sport England for funding this project through the Lottery-supported Get Healthy, Get Active portfolio.

Contributions: HR, NC, conceived the study, led the consultative meetings; drafted the system maps and led on the drafting of the paper; NC, conducted the qualitative fieldwork; DR, oversaw the intervention programme, organised the consultative meetings and drafted sections of the paper; MF, assisted with the intervention programme and led the ethics application; $\mathrm{CB}$, chaired the consultative meetings and oversaw the study. All authors provided input to and approved the final manuscript.

Ethics approval and consent to participate: The study was approved by the University of Derby Human Sciences Research Ethics Committee on 22nd March 2018 (Ethics Ref No: 25-1718$\mathrm{CBs}$ ). Written informed consent to participate was obtained from all participants, who were informed that their responses would be published.

Consent for publication: All participants in the research gave consent to publication.

Availability of data and material: The transcripts of interviews generated and/or analysed during the current study are not publicly available due to confidentiality agreements, but anonymised versions are available from the corresponding author on reasonable request.

Competing interests: The authors declare that they have no competing interests.

Funding: The project was funded by Sport England. The funders did not influence the study in any way: Sport England had no role in the design of the study, data collection, data analysis, data interpretation, or writing the manuscript.

Received for publication: 11 March 2020.

Accepted for publication: 14 July 2020.

oCopyright: the Author(s), 2020

Licensee PAGEPress, Italy

Journal of Public Health Research 2020;9:1759

doi:10.4081/jphr:2020.1759

This work is licensed under a Creative Commons Attribution NonCommercial 4.0 License (CC BY-NC 4.0). 


\section{References}

1. Sallis JF, Bull F, Guthold R, et al. Progress in physical activity over the Olympic quadrennium. Lancet 2016;388:1325-36.

2. Bauman A, Merom D, Bull FC, et al. Updating the evidence for physical activity: Summative reviews of the epidemiological evidence, prevalence, and interventions to promote "active aging". Gerontologist 2016;56:S268-80.

3. Hallal PC, Andersen LB, Bull FC, et al. Global physical activity levels: surveillance progress, pitfalls, and prospects. Lancet 2012;380:247-57.

4. Chau J, Chey T, Burks-Young S, et al. Trends in prevalence of leisure time physical activity and inactivity: results from Australian National Health Surveys 1989 to 2011. Aust N Z J Public Health 2017;41:617-24.

5. Heath GW, Parra DC, Sarmiento OLet al. Evidence-based intervention in physical activity: lessons from around the world. Lancet 2012;380:272-81.

6. Reis RS, Salvo D, Ogilvie D, et al. Scaling up physical activity interventions worldwide: stepping up to larger and smarter approaches to get people moving. Lancet 2016;388:1337-48.

7. Bronfenbrenner U, Ceci SJ. Nature-nurture reconceptualized in developmental perspective: A bioecological mode. Psychol Rev 1994;101:568-86.

8. Dahlgren G WM. Tackling inequalities in health: what can we learn from what has been tried? Working paper prepared for the King's Fund International Seminar on Tackling Inequalities in Health. London: King's Fund 1993.

9. Sallis JF ON, Fisher EB. Ecological models of health behavior. In K Glanz, BK Rimer and K Viswanath, editors. Health behavior and health education: Theory, research, and practice. San Francisco: Jossey-Bass; 2008. p. 465-85.

10. Pringle A, Gilson N, McKenna J, Cooke C. An evaluation of the Local Exercise Action Pilots and impact on moderate physical activity. Health Educ J 2009;68:179-85.

11. Cavill N, Muller L, Mulhall C, et al. Cycling Demonstration Towns: Surveys of cycling and physical activity 2006 to 2009 . London. Cycling England. 2009. Availabe from: http://www.cavill.net/app/media/documents/pages/30/cdtssurv eysofcyclingandphysicalactivity200609.pdf

12. Friel S, Pescud M, Malbon E, et al. Using systems science to understand the determinants of inequities in healthy eating. PLoS One 2017;12:e0188872.

13. Government Office for Science, Department of Health and Social Care. Tackling Obesities: Future Choices. London; 2007. Available from: https://www.gov.uk/government/collections/tackling-obesities-future-choices

14. Rutter H, Savona N, Glonti K, et al. The need for a complex systems model of evidence for public health. Lancet 2017;390:2602-4.
15. Rutter H, Cavill N, Bauman A, Bull F. Using a systems approach to support the development of global and national actions on physical activity. B World Health Organ 2019;97:162-5. doi: 10.2471/BLT.18.220533.

16. Allender S, Owen B, Kuhlberg J, et al. A community based systems diagram of obesity causes. PLoS One 2015; 10:e0129683.

17. Hovmand P. Community based system dynamics. New York: Springer; 2014.

18. Puska P. Why did North Karelia-Finland work?: Is it transferrable? Glob Heart 2016;11:387-91.

19. Luepker RV, Murray DM, Jacobs DR, Jr., Met al. Community education for cardiovascular disease prevention: risk factor changes in the Minnesota Heart Health Program. Am J Public Health 1994;84:1383-93.

20. Troelson J. Odense: Denmark's National Cycle City. 2005.

21. Sloman L, Cairns S, Goodman A, et al. Meta-analysis of outcomes of investment in the 12 local sustainable transport fund large projects: Final report to Department for Transport. 2017. Available from: https://assets.publishing.service.gov.uk/government/uploads/system/uploads/attachment_data/file/738267 /meta-analysis-of-lstf-large-projects-final-report.pdf

22. Goodwin DM, Cummins S, Sautkina E, et al. The role and status of evidence and innovation in the healthy towns programme in England: a qualitative stakeholder interview study. J Epidemiol Community Health 2013;67:106-12.

23. Sautkina E, Goodwin D, Jones A, et al. Lost in translation? Theory, policy and practice in systems-based environmental approaches to obesity prevention in the Healthy Towns programme in England. Health Place 2014;29:60-6.

24. Sport England. Local delivery pilots - the story so far 2019. Available from: https:/www.sportengland.org/our-work/localdelivery-pilots-community-of-learning/local-delivery-pilotsthe-story-so-far/

25. Bauman AE, Reis RS, Sallis JF, et al. Correlates of physical activity: why are some people physically active and others not? Lancet 2012;380:258-71.

26. Choi J, Lee M, Lee JK, et al. Correlates associated with participation in physical activity among adults: a systematic review of reviews and update. BMC Public Health 2017;17:356.

27. Barnett DW, Barnett A, Nathan A, et al. Built environmental correlates of older adults' total physical activity and walking: a systematic review and meta-analysis. Int J Behav Nutr Phys Act 2017;14:103.

28. Sport England. Active Lives Surveys 2019. Available from: https://www.sportengland.org/research/active-lives-survey/

29. Meadows DH. Project. Leverage points. Places to intervene in a system. Hartland: The Sustainability Institute; 1999. Available from: http://donellameadows.org/wp-content/userfiles/Leverage_Points.pdf 